\title{
A TERNARY OPERATION IN DISTRIBUTIVE LATTICES
}

\section{GARRETT BIRKHOFF AND S. A. KISS}

It can be easily seen that the graph $\left[1\right.$, p. 9], ${ }^{1}$ of the Boolean algebra $B^{n}$ of $2^{n}$ elements (consisting of the vertices and edges of an $n$-cube) has $2^{n}(n !)$ "link-automorphisms," whereas $B^{n}$ has only $(n !)$ lattice-automorphisms. In an unpublished book, ${ }^{2}$ one of us has developed new operations in $B^{n}$ and other distributive lattices, which admit such a wider group of invariance. The purpose of this note is to show the role of the symmetric and self-dual ternary operation $[1$, p. 74]

$$
\begin{aligned}
(a, b, c) & =(a \cap b) \cup(b \cap c) \cup(c \cap a) \\
& =(a \cup b) \cap(b \cup c) \cap(c \cup a)
\end{aligned}
$$

in a general distributive lattice $L$, with reference to the wider group of symmetries which it admits.

THEOREM 1. In any metric distributive lattice [1, p. 41], the following conditions are equivalent: (i) $a \cap b \leqq x \leqq a \cup b$, (ii) $|a-x|+|x-b|$ $=|a-b|$, (iii) $(a, x, b)=x$.

Proof. V. Glivenko [3, p. 819, Theorem V] has shown the equivalence of (i) and (ii); condition (i) says that $x$ is metrically "between" $a$ and $b$ in the sense of Menger. But now if $a \cap b \leqq x \leqq a \cup b$, then $(a, x, b)=(a \cap b) \cup(b \cap x) \cup(x \cap a)=(a \cap b) \cup[x \cap(a \cup b)]=x$. Conversely, if $(a, x, b)=x$, then

$$
x=(a \cap b) \cup(b \cap x) \cup(x \cap a) \geqq a \cap b,
$$

and dually, $x \leqq a \cup b$. Hence (i) and (iii) are equivalent.

Definition. The segment joining $a$ and $b$ is the set of $x$ satisfying any (hence all) of the conditions of Theorem 1 (cf. Duthie [4]); we denote it $[a, b]$.

THEOREM 2. The element $(a, b, c)$ is the intersection of the sets $[a, b][b, c][c, a]$.

Proof. This is obvious from condition (i) and formula (1).

\section{Corollary 1. The element $(a, b, c)$ minimizes}

Received by the editors October 21, 1946, and, in revised form, January 16, 1947.

1 Numbers in brackets refer to the bibliography at the end of the paper.

$2 \mathrm{~S}$. A. Kiss, Transformations on lattices and structures of logic, Bull. Amer. Math. Soc. Abstract 52-1-4. 


$$
|x-a|+|x-b|+|x-c| \text {. }
$$

Proof. In fact, $|x-a|+|x-b| \geqq|a-b|$ and symmetrically. Adding, we get for all $x$

$$
\begin{aligned}
2\{|x-a|+|x-b|+|x-c|\} & \\
& \geqq|a-b|+|b-c|+|c-a|
\end{aligned}
$$

By Theorems 1 and 2, equality holds if and only if $x=(a, b, c)$.

Since $(a, b, c)$ can be defined in terms of distance and $a, b, c$, we get, further, the following corollary.

CoRollary 2. Any isometry of a metric distributive lattice preserves the operation $(a, b, c)$.

In neither nondistributive lattice of five elements, does every triple of elements $a, b, c$, determine a unique $x$ minimizing $|x-a|+|x-b|$ $+|x-c|$; it would be interesting to know what the lattices are in which this "midpoint" is uniquely determined.

By using a weighted dimension function [1, Theorem 3.9] on $B^{2}$, we can find easily lattice-automorphisms which are not isometries. However, in the finite-dimensional case, we can obtain a converse to the preceding corollary.

We define distance in the graph of a finite-dimensional lattice in the usual way, by making all segments have length one. Thus if $d[x]$ is the number of "links" in the longest chain joining $O$ and $x$, $|x-y|=d[x \cup y]-d[x \cap y]$. This makes $L$ a metric lattice $[1$, Theorem 3.9].

THEOREM 3. The isometries of the graph of a finite-dimensional distributive lattice $L$ are precisely the automorphisms with respect to the ternary operation $(a, b, c)$.

Proof. Corollary 2 above proves one half. To prove the converse, since any isometry carries "linked" elements into "linked" elements, it suffices to note the following lemma.

Lemma. In $L, a$ and $b$ are "linked" (that is, $a$ covers $b$ or $b$ covers $a$ ) if and only if $(a, x, b)=a$ or $b$ for all $x$.

Proof. By Theorem $1,(a, x, b)=a$ or $b$ for all $x$ if and only if $[a, b]$ consists only of $a$ and $b$ - that is, if and only if $a$ and $b$ are equal or linked.

Definition. We shall call elements $a, a^{\prime}$ of a distributive lattice complementary if and only if $\left(a, x, a^{\prime}\right)=x$ for all $x$.

It is easily seen that a given $a$ can have only one complement. 
For if it has two, say $a^{\prime}$ and $a^{\prime \prime}$, then $a^{\prime \prime}=\left(a, a^{\prime \prime}, a^{\prime}\right)=\left(a, a^{\prime}, a^{\prime \prime}\right)=a^{\prime}$, for any symmetric ternary operation. It is also easily seen that if $a$ and $a^{\prime}$ are complementary, then we have a "double algebra" with an "extreme pair"; $;$ we denote $x \cap y=(x, a, y)$ and $x \cup y=\left(x, a^{\prime}, y\right)$, and get a distributive lattice.

More generally, the $2^{n}$ mutually distributive operations introduced by one of us $^{2}$ in $B^{n}$ are the operations $(x, a, y)$, one for each of the $2^{n}$ different elements $a$ of $B^{n}$.

Added January 15, 1947. It may be shown that distributive lattices can be defined in terms of the ternary operation $(a, b, c)$, by postulating

$$
\begin{array}{rlr}
(0, a, I) & =a & \text { for a fixed pair } O, I, \\
(a, b, a) & =a, & \\
(a, b, c) & =(b, a, c)=(b, c, a) & \text { (symmetry), } \\
((a, b, c), d, e) & =((a, d, e), b,(c, d, e)) . &
\end{array}
$$

The proof is straightforward, if we try to prove the right known set of postulates. ${ }^{4}$ The greatest trouble comes in proving that $(a, b, c)$ is actually defined by (1) in the distributive lattice defined by the binary operations $a \cap b \equiv(a, 0, b)$ and $a \cup b \equiv(a, I, b)$. This trouble is resolved by using Theorem 2 above. Law (3.4), which contains both ordinary distributive laws as special cases, may be proved by direct substitution.

The structure of the group of automorphisms of $B^{n}$ with respect to the ternary operation $(a, b, c)$ is of interest for its own sake, and because it is the structure of the group of symmetries of the $n$-cube.

For any fixed $a$, the correspondence

$$
x \rightarrow\left(x \cap a^{\prime}\right) \cup\left(x^{\prime} \cap a\right)=x+a
$$

(here + denotes addition in the additive group of the corresponding Boolean ring) is an automorphism for $(a, b, c)$; this defines a simply transitive, elementary Abelian subgroup of order $2^{n}$, of symmetries of the $n$-cube; the case $a=I, x \rightarrow x^{\prime}$ gives the "antipodal reflection" generating the center of the group. We also have the subgroup "of

${ }^{3}$ Cf. M. H. A. Newman, A characterization of Boolean lattices and rings, J. London Math. Soc. vol. 16 (1941) p. 257.

${ }^{4}$ Namely, those of p. 7 of Distributive postulates for systems like Boolean algebras, by G. D. Birkhoff and G. Birkhoff, Trans. Amer. Math. Soc. vol. 60 (1946) pp. 3-11. It might be possible to base postulates on the conditions of $\$ \S 8-10$ of E. Pitcher and M. F. Smiley, Transitivities of betweenness, Trans. Amer. Math. Soc. vol. 52 (1942) pp. 95-114. 
stability" leaving the vertex $O$ fixed, and consisting of the latticeautomorphisms of $B^{n}$. The entire group is the product of these two subgroups.

There is an obvious analogy between the ternary operation (1) and the ternary operation $a b^{-1} c$ of a group $G$; in the latter case, the group of automorphisms for $a b^{-1} c$ is the holomorph ${ }^{5}$ of $G$. This similarly is the product of the subgroup of group-automorphisms and a simply transitive subgroup of right-translations $x \rightarrow x a$. It would be interesting to extend our ternary operation to Newman's "double systems" (reference of footnote 2).

However, it should be noted that in doing this, we should not use the ternary operation $a+b+c$ of Baer-Certaine. For with respect to this, $B^{n}$ has $2^{n}\left(2^{n}-1\right) \cdots\left(2^{n}-2^{n-1}\right)$ automorphisms; hence we cannot even define $(a, b, c)$ in terms of it.

\section{BIBLIOGRAPHY}

1. Garrett Birkhoff, Lattice theory, Amer. Math. Soc. Colloquium Publications, vol. 25, New York, 1940.

2. W. D. Duthie, Segments of ordered sets, Trans. Amer. Math. Soc. vol. 51 (1942) pp. 1-14.

3. V. Glivenko, Géometrie des systèmes de choses normées, Amer. J. Math. vol. 58 (1936) pp. 799-828.

4. - Contribution à l'études des systèmes de choses normées, ibid. vol. 59 (1937) pp. 941-956.

HARVARD UNIVERSITY AND

Standard Oil Development Company

${ }^{5} \mathrm{~J}$. Certaine, The ternary operation $(a b c)=a b^{-1} c$ of a group, Bull. Amer. Math. Soc. vol. 49 (1943) pp. 869-877; this operation had previously been discussed by R. Baer. It satisfies $(a a b)=b$, instead of $(a a b)=a$. 